\title{
A NOTE ON AN UNSTEADY FLOW OF AN OLDROYD-B FLUID
}

\author{
C. FETECAU AND K. KANNAN \\ Received 5 March 2005 and in revised form 5 June 2005
}

The velocity fields and the associated tangential tractions corresponding to the flow induced by a flat plate that applies a specified stress in an Oldroyd-B fluid are determined by means of Fourier cosine transforms. The solutions corresponding to a Maxwell, secondgrade, and Navier-Stokes fluid appear as limiting cases of the solutions established here.

\section{Introduction}

The laminar flow of a number of fluids such as polymeric liquids, food products, paints, slurries, foams, and so forth cannot be adequately described by the help of the classical linearly viscous Newtonian model. Thus, there is a need to have at hand an arsenal of non-Newtonian fluid models and over the past several decades a variety of models have been developed. While the fluids of the differential type can explain, for instance, normal stress differences in a simple shear flow, shear thinning/shear thickening, and nonlinear creep, characteristics exhibited by some non-Newtonian fluids, they cannot describe the stress relaxation exhibited by certain liquids. Rate-type fluid models can be constructed that can describe stress relaxation, nonlinear creep, shear thinning/shear thickening, and normal stress differences in simple shear flows. Maxwell [8] developed the first rate-type one-dimensional model that could describe stress relaxation. Another important study of rate-type fluids is due to Burgers [2] who developed a one-dimensional model that has proved useful in describing the response of a class of geomaterials. The model due to Burgers includes the one-dimensional version of a popular rate-type model due to Oldroyd, namely, the Oldroyd-B model. Oldroyd [9] was the first to develop systematically three-dimensional rate-type models that met the requirements of frame indifference. While the Oldroyd-B model can describe stress relaxation and normal stress differences in a simple shear flow, it is incapable of describing shear thinning/shear thickening. It can however be easily generalized to describe shear thinning/thickening. Here, we study the flow of an Oldroyd-B fluid due to an impulsively started plate and we obtain an exact solution for the velocity field and stress in the form of a definite integral. Such solutions in addition to providing a solution to an interesting problem can also be used to check 
the accuracy of numerical schemes that are developed for complicated flows involving such fluids.

Waters and King [13] studied the start-up Poiseuille flow of an integral-type Oldroyd-B fluid in a straight circular tube and its decay from the steady-state condition when the pressure gradient is removed. The exact solution was obtained using the Laplace transform method. Since an integral form of Oldroyd-B fluid model is used, only one initial condition is required for this unsteady problem. Unsteady, pressure-driven flow of a classical Maxwell fluid in a pipe was studied by Rahaman and Ramkissoon [10]. Exact solutions were obtained as an infinite Fourier-Bessel series for different functional forms for the pressure gradients. The solutions exhibited a boundary layer for certain values of material parameters. Wood [14] considered the start-up of a helical flow of an Oldroyd-B fluid in a cylindrical annulus and obtained the results of Rahaman and Ramkissoon [10] as a degenerate case. Wood [14] obtained the velocity field by using an infinite FourierBessel series. Hayat et al. [7] obtained periodic solution to the magnetohydrodynamic flow of an Oldroyd-B fluid using Fourier transform methods and recently Erdogan [4] has studied the dynamics of an unsteady vortex in a second-grade fluid with zero initial conditions. The solution was obtained as a definite integral by employing Hankel transforms.

\section{Governing equations}

An incompressible Oldroyd-B fluid is characterized by the following constitutive equations (Oldroyd [9]):

$$
\begin{aligned}
\mathbf{T} & =-p \mathbf{I}+\mathbf{S}, \\
\mathbf{S}+\lambda\left(\dot{\mathbf{S}}-\mathbf{L S}-\mathbf{S L}^{T}\right) & =\mu\left[\mathbf{A}+\lambda_{r}\left(\dot{\mathbf{A}}-\mathbf{L} \mathbf{A}-\mathbf{A L}^{T}\right)\right],
\end{aligned}
$$

where $\mathbf{T}$ is the Cauchy stress tensor, $\mathbf{S}$ is the extra stress tensor, $-p \mathbf{I}$ denotes the indeterminate spherical stress due to the constraint of incompressibility, $\mathrm{L}$ is the velocity gradient tensor, $\mathbf{A}=\mathbf{L}+\mathbf{L}^{T}$ is the first Rivlin-Ericksen tensor, $\lambda$ and $\lambda_{r}$ are the relaxation and retardation times, $\mu$ is the dynamic viscosity, and the superposed dot indicates the material time derivative. This model includes as special cases the Maxwell model and the linearly viscous fluid model. For the special class of motions considered here, the governing equations also include the equations of motion for the second-grade fluid.

Recently, Rajagopal and Srinivasa [11] have developed a thermodynamic framework for systematically developing rate-type viscoelastic fluid models. This framework has at its basis a proper choice for how the body stores energy and dissipates energy. Within the context of such a theory, they developed a generalization of the Oldroyd-B model, which when linearized appropriately reduces to the classical Oldroyd-B model. It follows that the Oldroyd-B model, whose material moduli are constants, stores energy like a linearized elastic solid. Different manners of storing and dissipating energy lead to different models, and practically all the popular rate-type models can be obtained by making 
special choices within the context of the framework developed by Rajagopal and Srinivasa [11]. A central idea behind the theory is that the model that is chosen amongst a class of possible candidates is the one that maximizes entropy production.

In the following analysis, we will consider an unidirectional flow whose velocity field, in Cartesian coordinates, is given by

$$
\mathbf{v}=v(y, t) \mathbf{i}
$$

where $\mathbf{i}$ is the unit vector along the $x$ coordinate direction. The above velocity field automatically satisfies the constraint of incompressibility. We will assume that the extra stress $\mathbf{S}$ depends only on $y$ and $t$, that is, $\mathbf{S}=\mathbf{S}(y, t)$. In the absence of body forces and pressure gradient along the $x$ coordinate direction, the balance of linear momentum reduces to

$$
\lambda \partial_{t}^{2} v(y, t)+\partial_{t} v(y, t)=v\left(1+\lambda_{r} \partial_{t}\right) \partial_{y}^{2} v(y, t)
$$

where $\nu=\mu / \rho$ is the kinematic viscosity of the fluid, $\rho$ is its constant density, and the subscripts $y$ and $t$ indicate partial differentiation with respect to the corresponding variables.

It would be appropriate to point out that a popular technique for studying such unsteady flows, namely, Laplace transforms, may be inappropriate for certain unsteady flow problems, unless certain compatibility is met (see Bandelli et al. [1]). This point cannot be overemphasized. Though the solution is obtained after enforcing the appropriate initial conditions, the solution so obtained fails to satisfy them! Of course, this depends on the specific unsteady problem under consideration. Here, we use an alternate transform method. We use finite Fourier transforms to carry out the analysis and the solution that is obtained satisfies all the initial and boundary conditions.

\section{Exact solutions}

Let us consider an incompressible Oldroyd-B fluid at rest, lying over an infinitely extended plate that coincides with the $(x, z)$-plane. Let us suppose that at time zero, a shear is applied to the plate and owing to the shear, the fluid is gradually moved. The governing equation is given by (2.3) and the boundary and initial conditions are (cf. Bandelli et al. [1])

$$
\begin{gathered}
\left(\nu+\alpha \partial_{t}\right) \partial_{y} v(y, t)=\frac{f}{\rho}=\frac{T_{x y}+\lambda \partial_{t} T_{x y}}{\rho} \text { at } y=0, t>0, \\
v(y, t), \partial_{y} v(y, t) \longrightarrow 0 \quad \text { for } y \longrightarrow \infty, t>0 \\
v(y, 0)=\partial_{t} v(y, 0)=0, \quad y>0
\end{gathered}
$$


where $f$ is the shear stress applied to the plate, $T_{x y}$ is the shear stress, and $\alpha=\nu \lambda_{r}$. In the limit $\lambda \rightarrow 0$, the equation reduces to that for a second-grade fluid (it is important to note that when $\lambda \rightarrow 0$, the Oldroyd-B model does not reduce to that of a second-grade fluid, it is only the equation that reduces to the corresponding equation for a second-grade fluid) and also when $\lambda \rightarrow 0$ and $\lambda_{r} \rightarrow 0$, that is, for a Navier-Stokes fluid, the boundary condition (3.1) reduces to a problem of constant stress at the boundary.

In order to solve this problem, we will use, as carried out by Erdogan [3] for the flow of a second-grade fluid and Fetecau et al. [5] for an Oldroyd-B fluid, the Fourier cosine transform. By multiplying (2.3) by $\sqrt{2 / \pi} \cos (y \xi)$, integrating over $y$ from 0 to $\infty$, and taking into account the boundary and initial conditions, that is, (3.1)-(3.3), we find that (cf. Sneddon [12, Section 3])

$$
\lambda \partial_{t}^{2} v_{c}(\xi, t)+\left(1+\alpha \xi^{2}\right) \partial_{t} v_{c}(\xi, t)+\nu \xi^{2} v_{c}(\xi, t)=-\sqrt{\frac{2}{\pi}} \frac{f}{\rho}, \quad \xi, t>0
$$

where the Fourier cosine transform $v_{c}(\xi, t)$ of $v(\xi, t)$ has to satisfy the initial conditions

$$
v_{c}(\xi, 0)=\partial_{t} v_{c}(\xi, 0)=0, \quad \xi>0
$$

The solution of the ordinary differential equation (3.4) with the initial conditions (3.5) is of the form

$$
\begin{aligned}
& v_{c}(\xi, t)=\sqrt{\frac{2}{\pi}} \frac{f}{\mu \xi^{2}}\left\{\frac{r_{2} \exp \left(r_{1} t\right)-r_{1} \exp \left(r_{2} t\right)}{r_{2}-r_{1}}-1\right\} \quad \text { if } \lambda<\lambda_{r}, \\
& v_{c}(\xi, t)=\sqrt{\frac{2}{\pi}} \frac{f}{\mu \xi^{2}}\left\{\frac{\alpha \xi^{2} \exp (-t / \lambda)-\exp \left(-\nu \xi^{2} t\right)}{\alpha \xi^{2}-1}-1\right\} \quad \text { if } \lambda=\lambda_{r}, \\
& v_{c}(\xi, t)=\left\{\sqrt{\frac{2}{\pi} \frac{f}{\mu \xi^{2}}\left[\frac{r_{2} \exp \left(r_{1} t\right)-r_{1} \exp \left(r_{2} t\right)}{r_{2}-r_{1}}-1\right], \quad \xi \in(0, a] \cup[b, \infty),}\right. \\
& \sqrt{\frac{2}{\pi}} \frac{f}{\mu \xi^{2}}\left\{\exp \left(-\frac{1+\alpha \xi^{2}}{2 \lambda} t\right)\left[\cos \left(\frac{\beta t}{2 \lambda}\right)+\frac{1+\alpha \xi^{2}}{\beta} \sin \left(\frac{\beta t}{2 \lambda}\right)\right]-1\right\}, \quad \xi \in(a, b),
\end{aligned}
$$

if $\lambda>\lambda_{r}$ and $\left(r_{1,2}=\left(-1+\alpha \xi^{2}\right) \pm \sqrt{\left(1+\alpha \xi^{2}\right)^{2}-4 \nu \lambda \xi^{2}}\right) /(2 \lambda), \beta=\sqrt{4 \nu \lambda \xi^{2}-\left(1+\alpha \xi^{2}\right)^{2}}, a=$ $1 /\left[\sqrt{\nu}\left(\sqrt{\lambda}+\sqrt{\lambda-\lambda_{r}}\right)\right]$, and $b=1 /\left[\sqrt{\nu}\left(\sqrt{\lambda}-\sqrt{\lambda-\lambda_{r}}\right)\right]$. 
Inverting (3.6) by means of Fourier's cosine formula (Sneddon [12]), we find that (here, we have also used the well-known result $\int_{0}^{\infty}\left(\sin ^{2}(y) / y^{2}\right) d y=\pi / 2$ (see, e.g., Grandshteyn and Ryzhik [6]))

$$
\begin{aligned}
v(y, t)= & \frac{f y}{\mu}-\frac{2 f}{\mu \pi} \int_{0}^{\infty}\left\{1-\frac{r_{2} \exp \left(r_{1} t\right)-r_{1} \exp \left(r_{2} t\right)}{r_{2}-r_{1}} \cos (y \xi)\right\} \frac{d \xi}{\xi^{2}} \quad \text { if } \lambda<\lambda_{r} \\
v(y, t)= & \frac{f y}{\mu}-\frac{2 f}{\mu \pi} \int_{0}^{\infty}\left\{1-\frac{\alpha \xi^{2} \exp (-t / \lambda)-\exp \left(-\nu \xi^{2} t\right)}{\alpha \xi^{2}-1} \cos (y \xi)\right\} \frac{d \xi}{\xi^{2}} \quad \text { if } \lambda=\lambda_{r} \\
v(y, t)= & \frac{f y}{\mu}-\frac{2 f}{\mu \pi} \int_{0}^{a}\left\{1-\frac{r_{2} \exp \left(r_{1} t\right)-r_{1} \exp \left(r_{2} t\right)}{r_{2}-r_{1}} \cos (y \xi)\right\} \frac{d \xi}{\xi^{2}} \\
& -\frac{2 f}{\mu \pi} \int_{a}^{b}\left\{1-\exp \left(-\frac{1+\alpha \xi^{2}}{2 \lambda} t\right)\left[\cos \left(\frac{\beta t}{2 \lambda}\right)+\frac{1+\alpha \xi^{2}}{\beta} \sin \left(\frac{\beta t}{2 \lambda}\right)\right] \cos (y \xi)\right\} \frac{d \xi}{\xi^{2}} \\
& -\frac{2 f}{\mu \pi} \int_{b}^{\infty}\left\{1-\frac{r_{2} \exp \left(r_{1} t\right)-r_{1} \exp \left(r_{2} t\right)}{r_{2}-r_{1}} \cos (y \xi)\right\} \frac{d \xi}{\xi^{2}} \quad \text { if } \lambda>\lambda_{r} .
\end{aligned}
$$

The extra stress $\mathbf{S}$ can be easily determined from $(2.1)_{2},(3.7)-(3.9)$, and the initial condition $\mathbf{S}(y, 0)=\mathbf{0}, y>0$, the fluid being at rest up to the moment $t=0$. Following Fetecau et al. [5], we have $S_{x z}=S_{y y}=S_{y z}=S_{z z}=0$ and

$$
\tau(y, t)=S_{x y}(y, t)=\frac{\mu}{\lambda} \exp \left(-\frac{t}{\lambda}\right) \int_{0}^{t} \exp \left(\frac{\tau}{\lambda}\right)\left(1+\lambda_{r} \partial_{\tau}\right) \partial_{y} v(y, \tau) d \tau .
$$

Introducing (3.7)-(3.9) into (3.10), we find that

$$
\begin{aligned}
\tau(y, t)= & f\left[1-\exp \left(-\frac{t}{\lambda}\right)\right]-\frac{2 f}{\lambda \pi} \int_{0}^{\infty} \frac{\exp \left(r_{2} t\right)-\exp \left(r_{1} t\right)}{r_{2}-r_{1}} \frac{\sin (y \xi)}{\xi} d \xi \quad \text { if } \lambda<\lambda_{r}, \\
\tau(y, t)= & f\left[1-\exp \left(-\frac{t}{\lambda}\right)\right]-\frac{2 f}{\pi} \int_{0}^{\infty} \frac{\exp \left(-\nu \xi^{2} t\right)-\exp (-t / \lambda) \sin (y \xi)}{\alpha \xi^{2}-1} d \xi \quad \text { if } \lambda=\lambda_{r}, \\
\tau(y, t)= & f\left[1-\exp \left(-\frac{t}{\lambda}\right)\right]-\frac{2 f}{\lambda \pi} \int_{0}^{a} \frac{\exp \left(r_{2} t\right)-\exp \left(r_{1} t\right) \frac{\sin (y \xi)}{r_{2}-r_{1}} d \xi}{\xi} \\
& -\frac{4 f}{\pi} \exp \left(-\frac{t}{2 \lambda}\right) \int_{a}^{b} \exp \left(-\frac{\alpha \xi^{2}}{2 \lambda} t\right) \sin \left(\frac{\beta t}{2 \lambda}\right) \frac{\sin (y \xi)}{\beta \xi} d \xi \\
& -\frac{2 f}{\lambda \pi} \int_{b}^{\infty} \frac{\exp \left(r_{2} t\right)-\exp \left(r_{1} t\right)}{r_{2}-r_{1}} \frac{\sin (y \xi)}{\xi} d \xi \quad \text { if } \lambda>\lambda_{r} .
\end{aligned}
$$


3190 A note on an unsteady flow of an Oldroyd-B fluid

\section{Limiting cases}

(1) Taking the limit of (3.7) and (3.11) as $\lambda \rightarrow 0$, we get the following solutions (the first of them being identical with equation (9) of Erdogan [3]):

$$
\begin{aligned}
& v(y, t)=\frac{f y}{\mu}-\frac{2 f}{\mu \pi} \int_{0}^{\infty} \frac{1}{\xi^{2}}\left\{1-\exp \left(-\frac{\nu \xi^{2}}{1+\alpha \xi^{2}} t\right) \cos (y \xi)\right\} d \xi, \\
& \tau(y, t)=f-\frac{2 f}{\pi} \int_{0}^{\infty} \frac{1}{1+\alpha \xi^{2}} \exp \left(-\frac{\nu \xi^{2}}{1+\alpha \xi^{2}} t\right) \frac{\sin (y \xi)}{\xi} d \xi
\end{aligned}
$$

which correspond to the case for a second-grade fluid.

(2) By letting $\lambda_{r} \rightarrow 0$ in (3.9) and (3.13), we find that

$$
\begin{aligned}
v(y, t)= & \frac{f y}{\mu}-\frac{2 f}{\mu \pi} \int_{0}^{1 /(2 \sqrt{\nu \lambda})}\left\{1-\frac{r_{4} \exp \left(r_{3} t\right)-r_{3} \exp \left(r_{4} t\right)}{r_{4}-r_{3}} \cos (y \xi)\right\} \frac{d \xi}{\xi^{2}} \\
& -\frac{2 f}{\mu \pi} \int_{1 /(2 \sqrt{\nu \lambda})}^{\infty}\left\{1-\exp \left(-\frac{t}{2 \lambda}\right)\left[\cos \left(\frac{\delta t}{2 \lambda}\right)+\frac{1}{\delta} \sin \left(\frac{\delta t}{2 \lambda}\right)\right] \cos (y \xi)\right\} \frac{d \xi}{\xi^{2}}, \\
\tau(y, t)= & f\left[1-\exp \left(-\frac{t}{\lambda}\right)\right]-\frac{2 f}{\lambda \pi} \int_{0}^{1 /(2 \sqrt{\nu \lambda})} \frac{\exp \left(r_{4} t\right)-\exp \left(r_{3} t\right)}{r_{4}-r_{3}} \frac{\sin (y \xi)}{\xi} d \xi \\
& -\frac{4 f}{\pi} \exp \left(-\frac{t}{2 \lambda}\right) \int_{1 /(2 \sqrt{\nu \lambda})}^{\infty} \frac{1}{\delta} \sin \left(\frac{\delta t}{2 \lambda}\right) \frac{\sin (y \xi)}{\xi} d \xi
\end{aligned}
$$

which are the solutions corresponding to a Maxwell fluid. Here $r_{3,4}=\left(-1 \pm \sqrt{1-4 \nu \lambda \xi^{2}}\right) /$ $(2 \lambda)$ and $\delta=\sqrt{4 \nu \lambda \xi^{2}-1}$.

(3) In the special case when both $\lambda_{r}$ and $\lambda \rightarrow 0$ in any one of (3.7)-(3.9) and (3.11)(3.13) or $\lambda_{r} \rightarrow 0$ in (4.1) or $\lambda \rightarrow 0$ in (4.2), the solutions are given by

$$
\begin{aligned}
& v(y, t)=\frac{f y}{\mu}-\frac{2 f}{\mu \pi} \int_{0}^{\infty} \frac{1}{\xi^{2}}\left\{1-\exp \left(-\nu \xi^{2} t\right) \cos (y \xi)\right\} d \xi, \\
& \tau(y, t)=f-\frac{2 f}{\pi} \int_{0}^{\infty} \exp \left(-\nu \xi^{2} t\right) \frac{\sin (y \xi)}{\xi} d \xi,
\end{aligned}
$$


which correspond to the solution in the case of an incompressible Navier-Stokes fluid. The integral in (4.3), as specified in Erdogan [3], can be written in terms of the complimentary error function $\operatorname{erfc}(\cdot)$. The associated shear stress (4.4) can also be written (cf. Sneddon [12, the entry 6 of Table 5]) as follows:

$$
\tau(y, t)=f\left\{1-\operatorname{erf}\left(\frac{y}{2 \sqrt{v t}}\right)\right\}=f \operatorname{erfc}\left(\frac{y}{2 \sqrt{v t}}\right)
$$

where $\operatorname{erf}(\cdot)$ is the error function.

\section{Numerical results and conclusions}

In this work, we have established the velocity fields and the associated tangential tractions corresponding to the flow induced by a plate that applies a stress on the boundary of an Oldroyd-B fluid. The solution $v(y, t)$ given by (3.7)-(3.9) satisfies the partial differential equation (2.3) and all the imposed initial and boundary conditions. In the special cases when $\lambda \rightarrow 0, \lambda_{r} \rightarrow 0$ or both the characteristic time constants tend to zero, our solutions reduce to those corresponding to a second-grade, Maxwell, and NavierStokes fluid, respectively. The velocity and shear stress fields are nondimensionalized by defining

$$
\bar{y}=\sqrt{\frac{|f|}{\mu \nu}} y, \quad \bar{t}=\frac{|f|}{\mu} t,
$$

where $\bar{y}$ and $\bar{t}$ are the nondimensional counterparts of $y$ and $t$, respectively. By substituting the above expressions in any of (3.7)-(3.9), the nondimensionalized velocity $\bar{v}$ is $\sqrt{(\mu /|f| \nu)} v$ and $\bar{\xi}$ is $\sqrt{(\mu \nu /|f|)} \xi$. The nondimensional shear stress $\bar{\tau}$ is defined as $\tau /|f|$. Using (5.1) in (3.7)-(3.9), one can obtain the nondimensional relaxation and retardation times as $(|f| / \mu) \lambda$ and $(|f| / \mu) \lambda_{r}$, respectively. In Figure 5.1, we portray the nondimensional velocity field for the Oldroyd-B fluid and the various subclasses. We notice that, at all times, the Navier-Stokes solution has the highest velocity at the boundary. In contrast to the other liquids, the Maxwell liquid shows the sharpest decrease before the velocity becomes zero (see Figures 5.1(a) and 5.1(c)). For the particular set of constants chosen, in Figure 5.1(e), we notice that all the velocity profiles approach each other. In the plots (b), (d), and (f) of Figures 5.1 and 5.2, the stress at the boundary of the Maxwell and Oldroyd fluid changes with time and approaches the boundary stress of the Navier-Stokes and second-grade fluid as $t \rightarrow \infty$ (see (3.11)-(3.13)).In Figures 5.2(a), 5.2(b), and 5.2(c), the longer the time is, the more affected is the fluid farther away from the boundary. Referring to Figure 5.2, we notice that all but one of the velocity profiles approach each other, the last, which is the one with the largest relaxation time, takes a much longer time to 
3192 A note on an unsteady flow of an Oldroyd-B fluid

Nondimensional velocity, $|f| t / \mu=1$

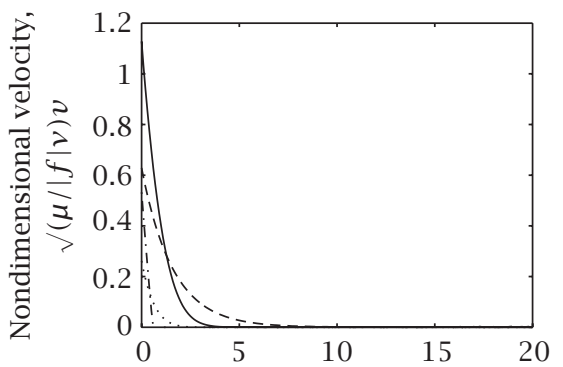

Nondimensional distance, $\sqrt{ }(|f| / \mu \nu) y$

(a)

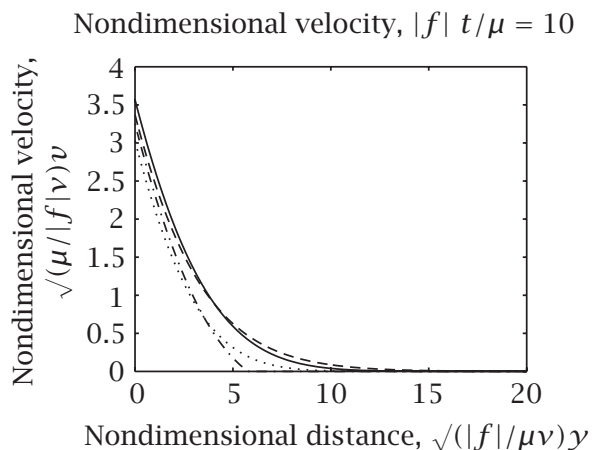

(c)

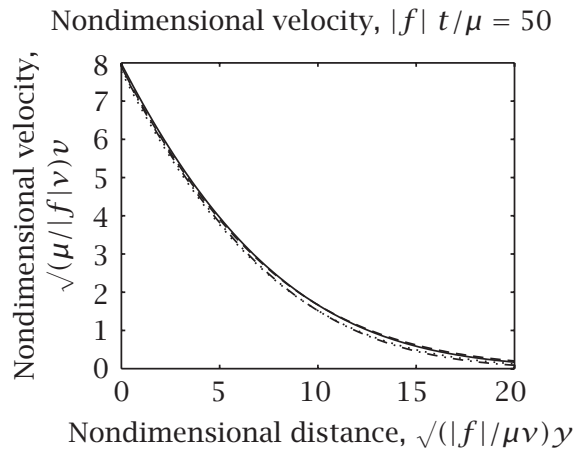

(e)
Nondimensional velocity, $|f| t / \mu=1$

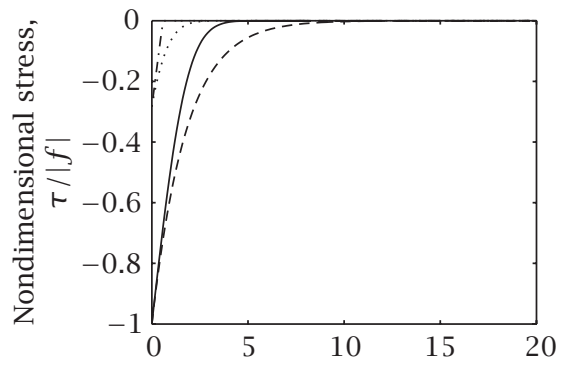

Nondimensional distance, $\sqrt{ }(|f| / \mu \nu) y$

(b)

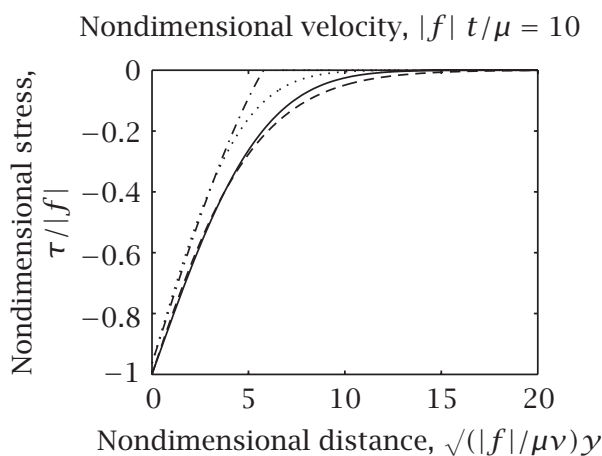

(d)

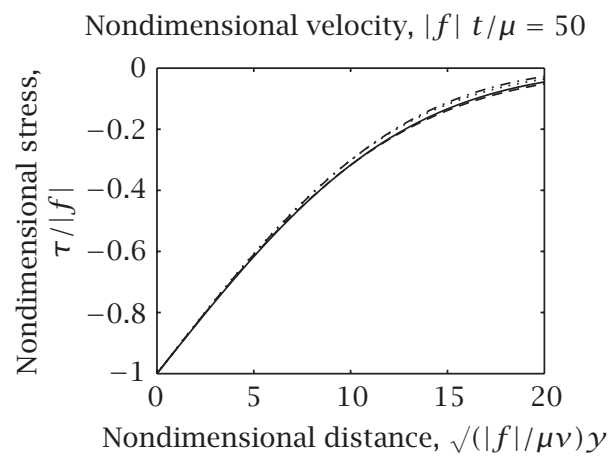

(f)

Figure 5.1 A portray of the nondimensional velocity field for the Oldroyd-B and the various subclasses. The solid curve denotes Navier-Stokes; the dashed curve denotes second-grade, $|f| \lambda_{r} / \mu=2$; the dashed dotted curve denotes Maxwell, $|f| \lambda / \mu=3$; the dotted curve denotes Oldroyd, $|f| \lambda_{r} / \mu=2$, $|f| \lambda / \mu=3$. 


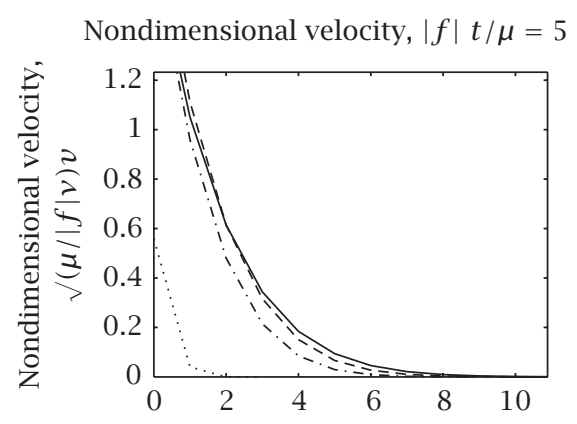

Nondimensional distance, $\sqrt{ }(|f| / \mu \nu) y$

(a)

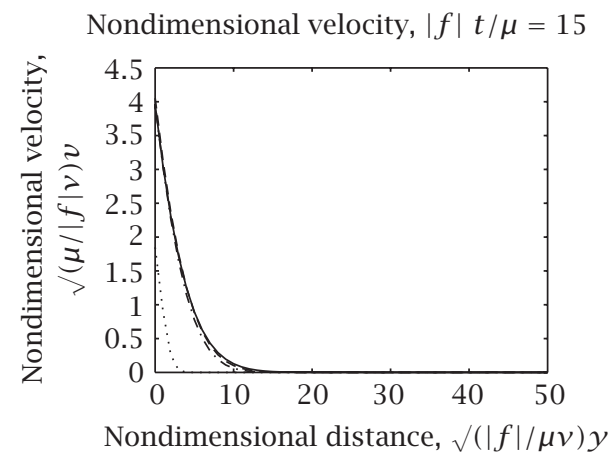

(c)

Nondimensional velocity, $|f| t / \mu=100$

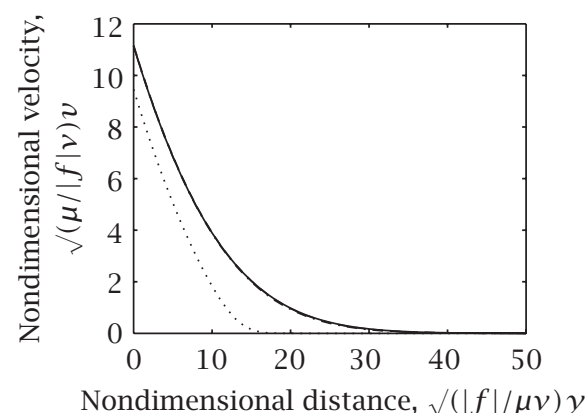

(e)
Nondimensional velocity, $|f| t / \mu=5$

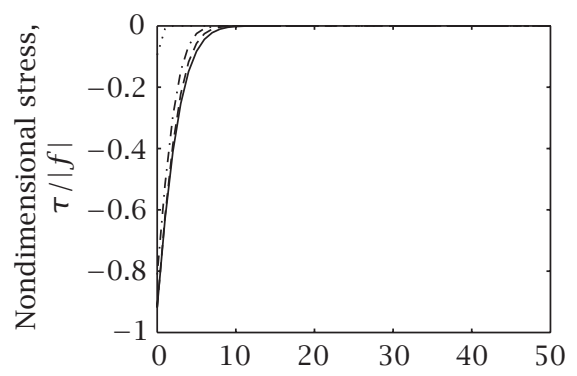

Nondimensional distance, $\sqrt{ }(|f| / \mu \nu) y$

(b)

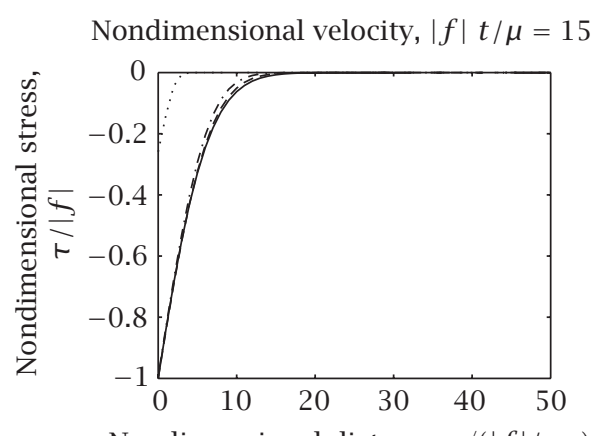

(d)

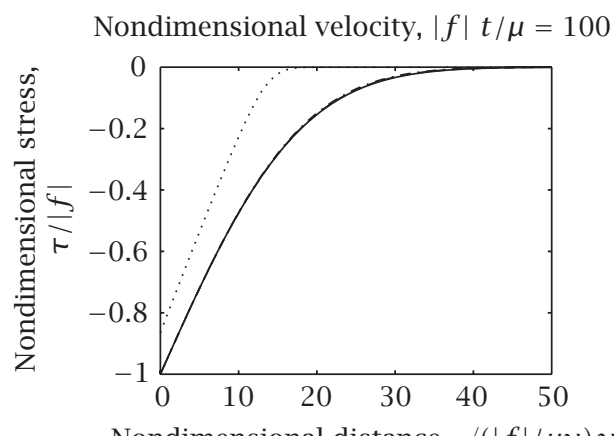

Nondimensional distance, $\sqrt{ }(|f| / \mu \nu) y$

(f)

Figure 5.2 A portray of the nondimensional velocity field for the Oldroyd-B fluid and the various subclasses. The solid curve denotes Oldroyd, $|f| \lambda_{r} / \mu=3,|f| \lambda / \mu=2$; the dashed curve denotes Oldroyd, $|f| \lambda_{r} / \mu=2,|f| \lambda / \mu=2$; the dashed dotted curve denotes Oldroyd, $|f| \lambda_{r} / \mu=2,|f| \lambda / \mu=3$; the dotted curve denotes Oldroyd, $|f| \lambda_{r} / \mu=2,|f| \lambda / \mu=50$. 
relax to the same asymptotic value of the others. Figures 5.2(b), 5.2(d), and 5.2(f) show the corresponding nondimensional shear stresses.

\section{References}

[1] R. Bandelli, K. R. Rajagopal, and G. P. Galdi, On some unsteady motions of fluids of second grade, Arch. Mech. (Arch. Mech. Stos.) 47 (1995), no. 4, 661-676.

[2] J. M. Burgers, Mechanical considerations-model systems-phenomenological theories of relaxation and viscosity, First report on viscosity and plasticity, Nordemann, New York, 1939.

[3] M. E. Erdogan, On unsteady motions of a second-order fluid over a plane wall, Internat. J. NonLinear Mech. 38 (2003), no. 7, 1045-1051.

[4] - Diffusion of a line vortex in a second-order fluid, Internat. J. Non-Linear Mech. 39 (2004), no. 3, 441-445.

[5] C. Fetecau, S. C. Prasad, and K. R. Rajagopal, Flow induced by a constantly accelerating plate in an Oldroyd-B fluid, submitted for publication, 2004.

[6] I. S. Gradshteyn and I. M. Ryzhik, Table of Integrals, Series, and Products, 5th ed., Academic Press, Massachusetts, 1994.

[7] T. Hayat, K. Hutter, S. Asghar, and A. M. Siddiqui, MHD flows of an Oldroyd-B fluid, Math. Comput. Modelling 36 (2002), no. 9-10, 987-995.

[8] J. C. Maxwell, On the dynamical theory of gases, Philos. Trans. R. Soc. London 157 (1867), 4988.

[9] J. G. Oldroyd, On the formulation of rheological equations of state, Proc. Roy. Soc. London Ser. A 200 (1950), no. 1063, 523-541.

[10] K. D. Rahaman and H. Ramkissoon, Unsteady axial viscoelastic pipe flows, J. Non-Newton Fluid Mech. 57 (1995), no. 1, 27-38.

[11] K. R. Rajagopal and A. R. Srinivasa, A thermodynamic frame work for rate type fluid models, J. Non-Newton Fluid Mech. 88 (2000), no. 3, 207-227.

[12] I. N. Sneddon, Functional Analysis, Encyclopedia of Physics, Springer, Berlin, 1955.

[13] N. D. Waters and M. J. King, The unsteady flow of an elastico-viscous liquid in a straight pipe of circular cross section, J. Phys. D: Appl. Phys. 4 (1971), no. 2, 204-211.

[14] W. P. Wood, Transient viscoelastic helical flows in pipes of circular and annular cross-section, J. Non-Newton Fluid Mech. 100 (2001), no. 1-3, 115-126.

C. Fetecau: Department of Mathematics, Technical University of Işai, 6600 Iaşi, Romania

E-mail address: fetecau@math.tuiasi.ro

K. Kannan: Department of Mechanical Engineering, Texas A\&M University, College Station, TX 77843, USA

E-mail address: krishna@neo.tamu.edu 


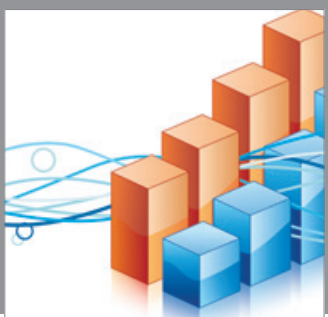

Advances in

Operations Research

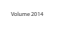

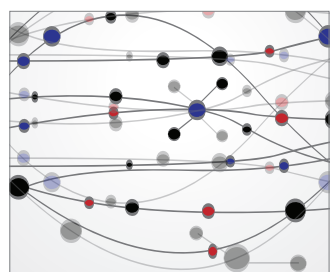

\section{The Scientific} World Journal
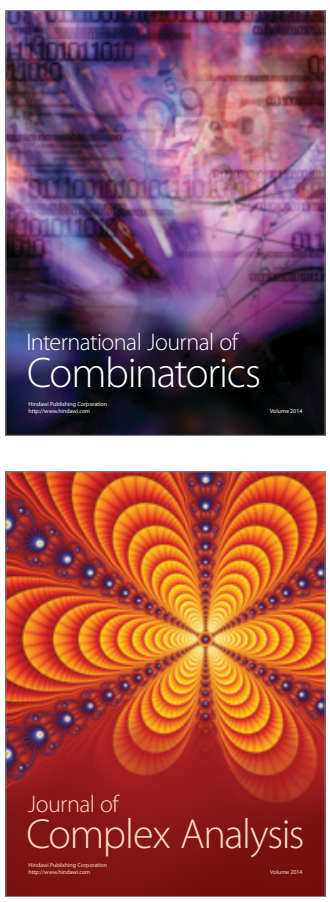

International Journal of

Mathematics and

Mathematical

Sciences
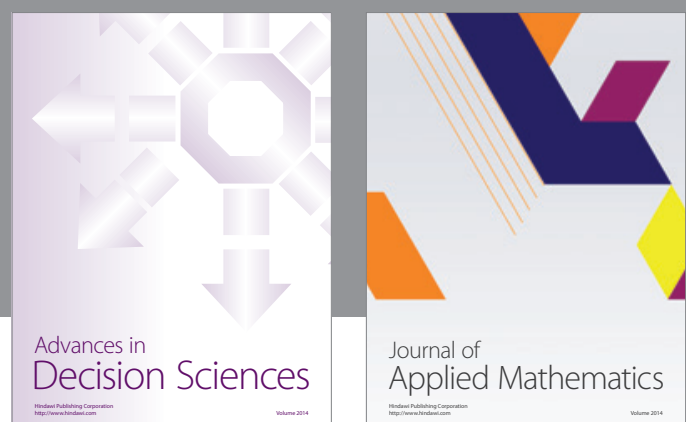

Journal of

Applied Mathematics
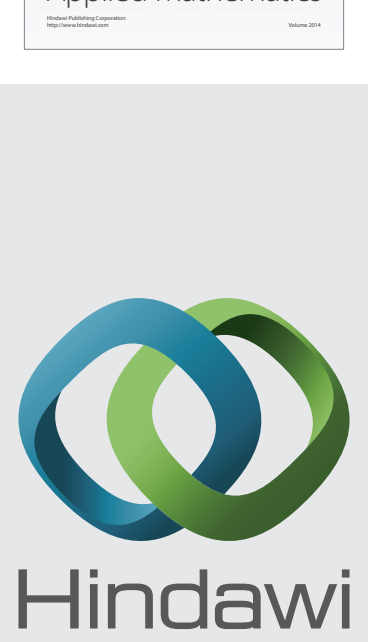

Submit your manuscripts at http://www.hindawi.com
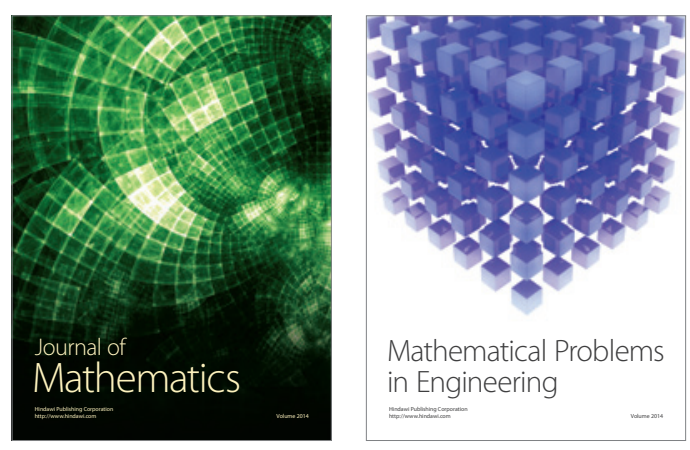

Mathematical Problems in Engineering
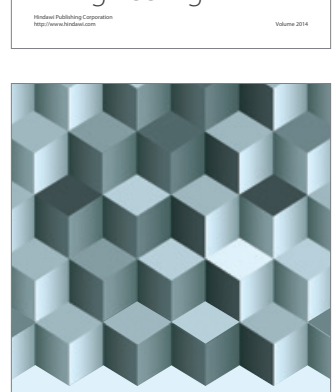

Journal of

Function Spaces
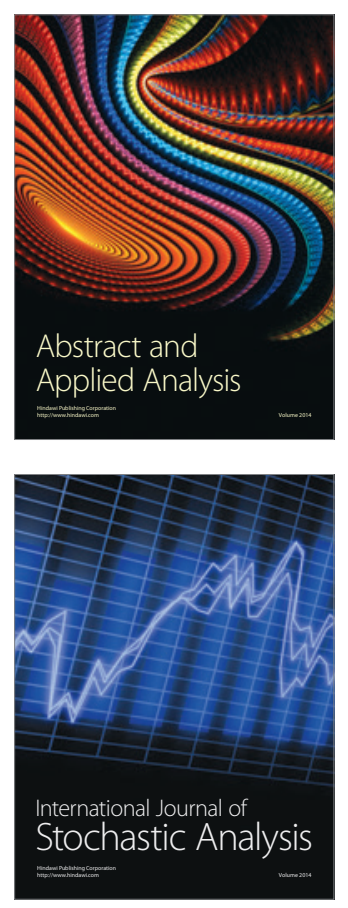

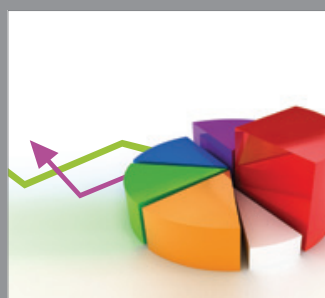

ournal of

Probability and Statistics

Promensencen
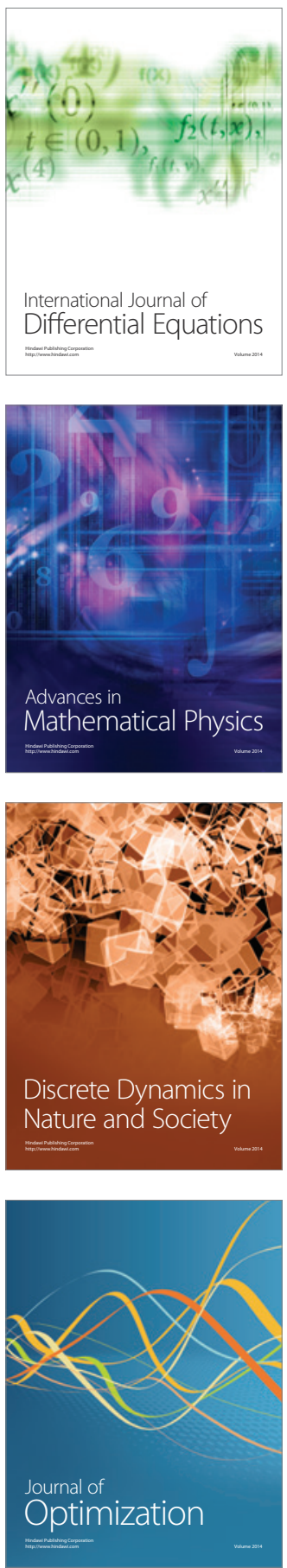